\title{
Digital Transactions in Real Estate Marketing
}

\author{
Jeffrey Shepard \\ Union Institute and University, Trustee of the University, Cincinnati, Ohio, United States of America
}

\section{Email address:}

shepard.jeffrey@gmail.com

\section{To cite this article:}

Jeffrey Shepard. Digital Transactions in Real Estate Marketing. Journal of World Economic Research. Vol. 9, No. 2, 2020 , pp. 110-114. doi: $10.11648 /$ j.jwer.20200902.15

Received: September 14, 2020; Accepted: October 22, 2020; Published: November 4, 2020

\begin{abstract}
Identity theft and tax fraud have been leading white-collar crimes for over a decade. Emerging trends have seen the rise in white-collar crime to now include deed theft. This type of theft marries identity theft and mortgage fraud, and it is robbing the hopes of many people who aspire to own a home. This whitepaper identifies how deed theft takes place through targeting homes with deceased owners, house flipping fraud and fake forensic auditors. It explores this evolved white-collar crime and its dangerous effects, while also revealing the best ways to mitigate the risk of deed theft. Furthermore, this paper provides the background of the crime and how the crime correlates with identify theft. Gaining control of this issue is challenging due to the existing infrastructure that was introduced in 1920. This leaves few country clerks with any response to the issue surrounding identity theft and mortgage fraud. To mitigate this challenge, devices have been developed to track the behaviour of people to ensure that their residences are not 'stolen'. Although these solutions are available, few individuals learn about deed theft so that the solutions can be used to protect themselves against it. This whitepaper presents DeedLock as the most effective solution to address the looming challenge of property theft.
\end{abstract}

Keywords: Deed Theft, DeedLock, Real Estate, Blockchain

\section{Introduction}

As technological advancements increasingly continue to penetrate the market, the property market climate and processes have begun to shift dramatically. Real estate has been one of the many sectors impacted explicitly by such shifts. Technology can make property sales more secure, especially to optimize workflows and provide cost-effective solutions [1]. Real estate deals involve significant amounts of capital, and structures that are not desirable may contribute to potentially high losses, particularly financially and by paperwork. By studying the fundamentals of real estate, it can be observed that nearly all deals are routine, that there is no formal paperwork with hard copy records being the rule, and that there are many parties involved [2]. Where more than two individuals make up most of the deal, inefficiencies are rife. Such shortfalls result in higher prices, ranging up to 10 percent of the overall amount of the purchase. For a fact, the period to close a contract is increased.

Blockchain infiltrates real estate, and the interest it puts onboard cannot be overlooked. It is anticipated that with Blockchain, all deeds, as well as mortgages, would soon be tokenized. It would support a wide range of players, including the government and banks. It is essential to be on the verge of this transition, and this is where the DeedLock put itself [3]. The first measure that DeedLock has taken to ensure that it will address this vacuum is to provide a robust technological framework for all companies who want to transition into blockchain technology. DeedLock is planned to combine with other systems to achieve outstanding performance. Buyers would profit from the opportunity to obtain a registry of their assets, which will also be exchanged with institutions such as governments, making it possible for all to get a straightforward understanding of their property. DeedLock would be based on a coin. In several specific programs, transfers may take place with tokens. This will encourage buyers, vendors, partnership members, and service providers to communicate with each other. With the tokens, it would be necessary to buy items on the network before the total supply has hit a sufficient threshold to ensure that the site can retain stability [4]. That is about 21 million. The ranking feature shall also be integrated into the website to enable sellers and buyers to complete their purchases. Via this method, excellent service providers can earn scores, allowing a network of service providers to become self-curing in the long run. 
DeedLock is also scalable, enabling the production of DeedLock network apps, which ensures that there is no need to think about outsourcing other resources [5]. The ICO is expected for DeedLock in the first quarter of 2018 and will have a hard limit of 35 million USD.

\section{How Deed Theft Works}

The offenders begin by paying careful attention to the obituaries to locate their victims. They are often searching for vacant or empty homes, professional homes, or rental property. They select the house as their goal, stealing identification by making false ID's, social security cards, and another document to enable the transfer of documents. If the house belongs to a deceased person, the descendant's name is forged on a deed to secure the title. With the ID in their possession, though a bogus one, comes a switch of deeds under which they register as actual landowners or personal officers. The proper authority shall convey the title to them, and the house will finally be theirs. Local 12 recorded such a case in Ohio, where an old brick house along Northside Hamilton Avenue was the focus of a court case alleging bribery and robbery. Real estate scammers prefer to own or sell a home, but in most situations, the latter is appropriate. They sell the land to an innocent third party in case something goes south. Such contracts contain a termination clause, which shall exonerate them of all responsibility after the transfer. The deed of the revocation implies that the purchaser will not promise that the title is safe and valid.

The FBI refers to deed theft as house robbery. In this sort of fraud, scammers rob your name and remove the deed to your home [6]. Over the past decade, billions of dollars have been destroyed as a consequence of the deed fraud - and this number continues to rise. Deed theft is a complex crime with a few turns and variants. When lost, it is a big obstacle for actual homeowners to get their properties back. This challenge goes beyond the pain of grappling with bringing the house back. It also requires a substantial cost of lawsuits. Initially considered a little established problem has now risen to near-crisis rates with a spike of up to 70 percent from one year to the next in Philadelphia [7].

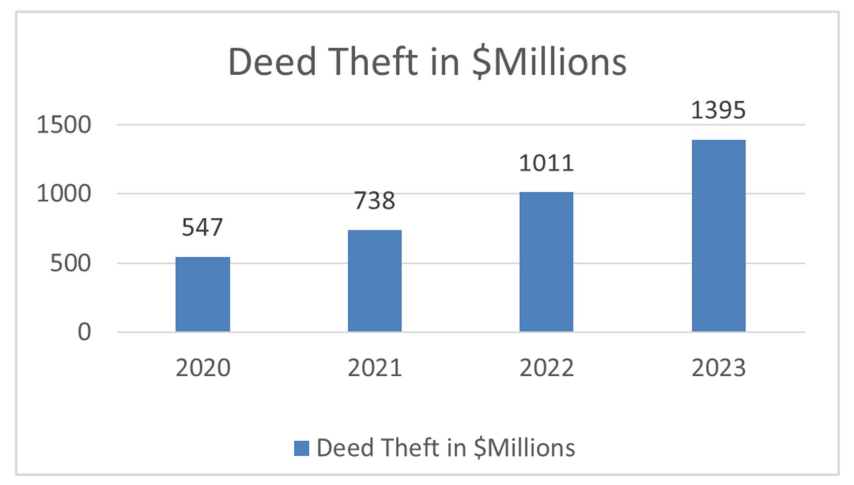

Figure 1. Trends in Property theft.

A glance at history shows a dramatic rise in deed theft, including damages of about 500 million USD over the last year.

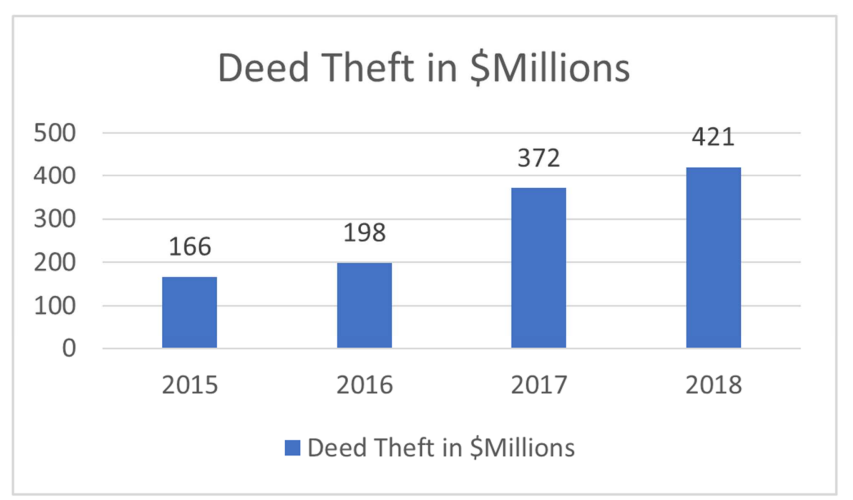

Figure 2. The Possible Future.

As fraud and theft detection in the property sector begin to intersect, the development of deed theft will rise in leaps and bounds. Under the present trend, deed stealing is estimated to cost more than 1 billion USD by 2022 .

\section{The Real Estate Industry - Challenges and Need for Transformation}

There are several players interested in real estate deals. This refers to domestic purchases, which are much more complicated when transacting through national boundaries. Take, for starters, transfers between East and West coasts [8]. The method is getting increasingly complicated as new regulations are being implemented, the regulatory structures of various states are specific, and all standards for paperwork, processes, and guidelines need to be updated [9]. There also exist legal threats and a need for knowledge of freedoms. In this, it must be borne in mind that specific industries are more forward-looking than others, particularly when it comes to administrative processes, which brings more difficulty to the operation.

\section{Challenges Facing the Real Estate Sector}

\subsection{Property Rights Enforcement and Documentation Inaccuracies}

For a title to be completed, in the U.S., the title provider would guarantee that there are no flaws in the title so that it can be applied on a mortgage. Across continents, this is achieved by national registration schemes [10]. Where the legal and administrative structure is weak, and a paper-based record-keeping mechanism is the standard, bribery, theft, and manipulation will delay the whole procedure. With Blockchain, it is feasible to obtain a digital certificate of possession of real estate property, which would make false advertising and forgeries a threat to the past [11]. It would be difficult to duplicate the names. Both sellers and buyers should be allowed to view any contact details via a shared 
ledger. The same extends to service suppliers. This can be achieved by a unique digital I.D. generated for both the seller and their consumer as well as the real estate.

\subsection{Full Process Transactional Challenges}

The very first step in every deal is for the consumer and the seller to decide on the amount. After this essential move has been accomplished, the rest may take a more challenging turn, particularly when it comes to closing the deal. Transactional problems include faulty paperwork, fragmented communications, standard processes; extra fees; administrative difficulties; pricing accountability; and exchange rates.

\subsection{Fraudulent and Inaccurate Mortgage-Related Documentation}

In 2008 , one of the hardest financial crisis years of this decade, more than 1,768 foreclosures happened attributable to faulty mortgage-related paperwork. As a result, more than half of the mortgage lenders do not have the correct paperwork to create a legal loan. Mortgage holder statements were over 1 billion USD more than what lenders would have owed [12].
Prof. Katherine Porter made this argument in a Texas Law Review report called "Error in Bankruptcy Mortgage Allegations." In her report, she established that this was a widespread phenomenon in the real estate industry that exposed vulnerabilities in current mortgage servicing. Records that have been reported as incomplete, such as itemization, promissory notes as well as security interest records.

\section{Introducing the DeedLock Model - a Transformational Solution}

The purpose of the DeedLock business strategy is to guarantee standardization, accountability, authentication, and verification of all transactions for real estate property. Last goals, include expense control, enhanced operating performance, decreased risk and increased precision. Through integrating Blockchain and non-blockchain technologies, both parties will profit [13]. The software is open to everyone as a cloud-based network that is suitable for purchasers around state lines.

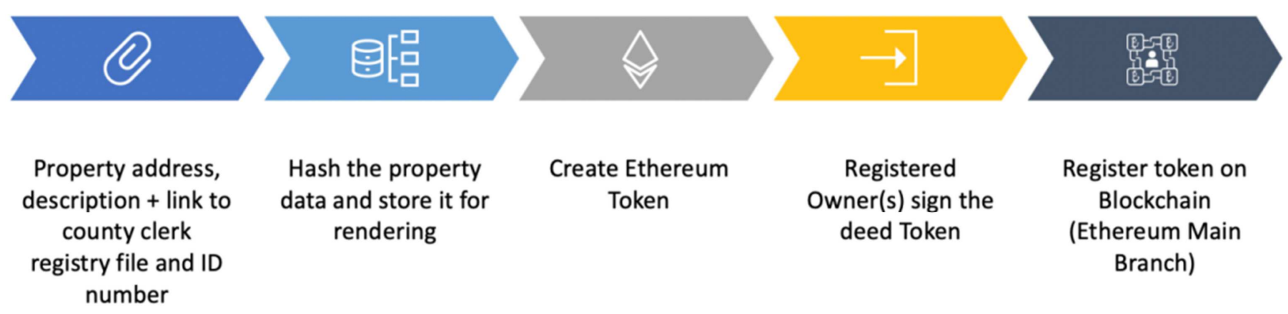

Figure 3. The DeedLock Mortgage or Deed Tokenization Process.

\subsection{Mortgage Tokens}

Property can be tokenized by bringing together material from the lender as well as a promissory notice. These two records include both the government register and the non-government register. With DeedLock, borrowers should assume that the documentation that has been done includes all the necessary records and that it is correct [14]. This allows them to mitigate their harm, which will enable them to impose their rights whenever payment defaults occur. Mortgage tokens can simplify the entire process of obtaining a mortgage, specifically with digital signatures [15]. When the mortgage has been repaid, the smart contract will be completed, and the bank's bond will be issued. When the property is offered on the black sector, there exists a risk that the transaction will not be listed in the government register. Tracking secondary mortgages on the market has been a struggle for some time, and MERS provides a workaround when paired with Blockchain.

\subsection{Deeds Tokens}

Property companies need an act for every deal to take effect. This legal instrument is usually a written document that establishes or contradicts value, right or property authenticated, attested, issued, and sealed in some jurisdictions. DeedLock is planned to digitize property documents and transform them into blockchain records. A formal summary of the properties will be initiated to order to move the properties [16]. Secondly, the names of the owner and the individual planning to buy the property are stated. Third, a signature is expected of the individual that will move the land. For DeedLock token to substitute the land, the first condition that is a legal definition of the land is met, rendering a clear transfer feasible [17]. It is since there are both Identification codes for the customer and the vendor to ensure that the second condition is achieved. Finally, the third criterion is fulfilled from the digital signatures, which have already been integrated into the application. Titles are often time-stamped, which fulfils the special condition that the certificate is notarized.

\section{The Role of Identity Tokens}

The main advantage of blockchain networks is that they are decentralized in design. Even with this, the connection between the public key of the person and their digital identification can be preserved. It can be used on the Internet using the https protocol used by governments to grant virtual identification cards that enable documents to be signed electronically $[18,19]$. Blockchain provides a compromise as 
the next phase in this cycle to create trust. Elliptic Curve Digital Signature Algorithm, also recognized as ECDSA, is shared key cryptography used for blockchains. Digitally, ECDSA will connect identification details to cryptographic signatures such that digital contracts and records can be checked. Someone has to use a private key, rendering it nearly difficult to duplicate. Both parties recognize the user's private key, which may also be verified against the public key [1]. For example, a consumer may have a private key, which is their personal and legal chain, and utilize their properties as a public key, tokenized on Blockchain. DeedLock aims to ensure that the databases are open and stable and that all information on the site can be completely checked. Thus, it should exploit infrastructure that has already been released to the public on blockchains, which are like bitcoin [20]. The key goal of DeedLock is to allow the use of the capabilities of Blockchain such that real estate transfers will become even easier. Eventually, it is anticipated that blockchain-based digital identity would make public notaries redundant when people continue to make use of legitimate digital signatures. In the long term, DeedLock must work directly with decision-makers to ensure that the legal legitimacy of ECDSA digital signatures is strengthened.

\section{Implementation of DeedLock}

Few DeedLock tokens are now accessible at a cheaper cost for customers to check out. However, this should change as the projected rise and ICO is predicted to develop within a few quarters. This will include a variety of stages [5]. Step 1 will be the pre-ICO and ICO phases in which all regulatory systems should be concerned with and partnerships to push DeedLock forward. Step 2 will be the focal point of REP deployment and improvement architecture [19]. It would be accompanied by a gradual opening as well as a commercial introduction. The final step would be the upgrade of existing information structures by collaborating with both city agencies and the blockchain network.

\subsection{Benefits}

Users should be able to encounter a few different advantages by opting to use DeedLock. Remote transfers are among the other advantages of DeedLock. Essentially, it has formed a relationship with e-signature providers that is successful in creating smart contracts. The advantage is the simplicity of transfers utilizing Cryptocurrencies. Bitcoin is a strong illustration of cryptocurrencies that stands for being widely distributed across various networks [5]. DeedLock shall work with a few payment services to ensure that cryptocurrencies can be translated into fiat currencies for purchases to occur. Besides, DeedLock offers the advantages of Automation, as nearly all procedure phases are automated, an aspect that allows for standardization to be even simpler, because records are exact and secure. DeedLock is a Reduced Cost Throughout as sellers and buyers can communicate with each other explicitly.

\subsection{Features}

So far, as the layout of DeedLock Token is involved, the network is operated by a native DeedLock functionality token. This single token allows both buyers and sellers to provide exposure to all the offerings that the network has to offer, including those from partnership members and other market providers [21, 22]. Other cryptocurrency firms would be partnered with DeedLock to add value, with DeedLock being used as a common currency to ensure smooth and easy use. App ratings are a core function of DeedLock, and ratings cannot be changed. This helps secure the consumers of the platforms.

\section{Blockchain Applications}

Blockchain implementations can also be built due to the versatility of DeedLock to reduce the costs of contracting related resources. This ensures that holders of tokens can benefit from an extra value [23]. The tokens that one earns to have feedback should be charged from the service provider, which has provided the report. This would mean that all service providers would be forced to keep any DeedLock tokens on their account. That would mean that there is a market for a larger quantity of tokens, as that will boost the value as more service companies want to utilize the network.

\section{Conclusion}

As technology advances, making use of available options such as Blockchain based solutions will be necessary across industries. In real estate, DeedLock addresses the issue of Deed Theft in an effective way. Real estate scammers have previously taken advantage of the numerous players that are required for a real estate deal to take place. Too many players lead to documentation inaccuracies and makes it challenging for any property rights enforcement to take place. Fraudulent and inaccurate mortgage related documentation have previously passed without detection or relevant action.

The research reveals that DeedLock brings everything together to ensure that there is accountability, authentication, standardization and verification of all real estate transactions. By tokenizing mortgages and deeds, it becomes easier to protect identities and centralize information. The DeedLock system works directly with decision makers, adding a level of legal legitimacy to all the secure features that it has. The future will require all service companies to utilize this blockchain based network, bringing down the cost of individually contracting service providers.

\section{References}

[1] Tunstall, M., Caplan, A., Prescott, N., \& Sandler, B. (2018). Real Property Transfers Ripe for Blockchain Disruption: Laws in the U.S. Must Follow the Technology. Includes Chapter News, 31 (1), 16. 
[2] Moosavi, V. (2017). Urban data streams and machine learning: a case of swiss real estate market. arXiv preprint arXiv: 1704.04979 .

[3] Fan, K., Sun, S., Yan, Z., Pan, Q., Li, H., \& Yang, Y. (2019). A blockchain-based clock synchronization scheme in IoT. Future Generation Computer Systems, 101, 524-533.

[4] Toradmalle, D., Muthukuru, J., \& Sathyanarayana, B. (2019). Certificateless and a provably-secure digital signature scheme based on an elliptic curve. International Journal of Electrical \& Computer Engineering (2088-8708), 9.

[5] Ismail, L., \& Materwala, H. (2019). A Review of Blockchain Architecture and Consensus Protocols: Use Cases, Challenges, and Solutions. Symmetry, 11 (10), 1198.

[6] FBI. (2008, 3 25). House Stealing. Retrieved from The FBI: https://archives.fbi.gov/archives/news/stories/2008/march/hou sestealing 032508

[7] Frost, M. (2019, April 8). Relief could be coming soon for victims of deed theft. Retrieved from Brooklyn Daily Eagle: https://brooklyneagle.com/articles/2019/04/08/relief-could-becoming-soon-for-victims-of-deed-theft/

[8] Johnson, D., Menezes, A., \& Vanstone, S. (2001). The elliptic curve digital signature algorithm (ECDSA). International journal of information security, 1 (1), 36-63.

[9] Kittur, A. S., \& Pais, A. R. (2017). Batch verification of digital signatures: approaches and challenges. Journal of information security and applications, 37, 15-27.

[10] Weider, D. Y., Gole, M., Prabhuswamy, N., Prakash, S., \& Shankaramurthy, V. G. (2016, June). An Approach to Design and Analyze the Framework for Preventing Cyberbullying. In 2016 IEEE International Conference on Services Computing (SCC) (pp. 864-867). IEEE.

[11] Schernthanner, H., Asche, H., Gonschorek, J., \& Scheele, L. (2020). Spatial modeling and geo-visualization of rental prices for real estate portals. In Cognitive Analytics: Concepts, Methodologies, Tools, and Applications (pp. 962-977). IGI Global.

[12] Sadikin, M. A., \& Wardhani, R. W. (2016, July). Implementation of RSA 2048-bit and AES 256-bit with digital signature for secure electronic health record application. In 2016 International Seminar on Intelligent Technology and Its Applications (ISITIA) (pp. 387-392). IEEE.
[13] Wadley, C. D., Dintenfass, K., Missouri, D. C., Wittkowski, A. C., Jones-McFadden, A. C., \& Thompson, A. F. (2017). U.S. Patent Application No. 14/851, 758.

[14] Li, Y., Wang, S., Yang, T., Pan, Q., \& Tang, J. (2017, June). Price Recommendation on Vacation Rental Websites. In Proceedings of the 2017 SIAM International Conference on Data Mining (pp. 399-407). Society for Industrial and Applied Mathematics.

[15] Pornin, T. (2013). Deterministic usage of the digital signature algorithm (DSA) and elliptic curve digital signature algorithm (ECDSA). Internet Engineering Task Force RFC, 6979, 1-79.

[16] Knoll, J., Groß, R., Schwanke, A., Rinn, B., \& Schreyer, M. (2018, June). Applying recommender approaches to the real estate e-commerce market. In International Conference on Innovations for Community Services (pp. 111-126). Springer, Cham.

[17] Levin, B. (2018). Potential for Cryptocurrency to Fund Investment in Sustainable Real Assets (Doctoral dissertation, Duke University).

[18] Morena, M., Truppi, T., Pavesi, A. S., Cia, G., Giannelli, J., \& Tavoni, M. (2020). Blockchain and real estate: Dopo di Noi project. Property Management.

[19] Pindado, G., \& Álvarez, J. M. Á. (2018). The GJ-STATIC securitization fund on property rentals. An instrument to help struggling real estate and construction companies in Spain. Aestimatio: The IEB International Journal of Finance, (16), $2-5$.

[20] Kundu, D. (2019). Blockchain and trust in a smart city. Environment and Urbanization ASIA, 10 (1), 31-43.

[21] Heil, D., Jenset, G., \& McGillivray, B. (2020). Extracting Keywords from Open-Ended Business Survey Questions. Journal of Data Mining \& Digital Humanities, 2020.

[22] Mills, D. J., \& Gotsill, D. (2020). Foreign Investors' Acceptance and Usage of PropTech in the Japanese Real Estate Market. In Transforming Japanese Business (pp. 197-208). Springer, Singapore.

[23] Farooq, S. M., Hussain, S. S., \& Ustun, T. S. (2019, March). Elliptic Curve Digital Signature Algorithm (ECDSA) Certificate-Based Authentication Scheme for Advanced Metering Infrastructure. In 2019 Innovations in Power and Advanced Computing Technologies (i-PACT) (Vol. 1, pp. 1-6). IEEE. 